le portiQue $\begin{array}{ll}\text { Le Portique } \\ \text { Revue de philosophie et de sciences humaines }\end{array}$

3| 1999

Technique et esthétique

\title{
Art et mécanique
}

\section{Pierre-Damien Huyghe}

\section{OpenEdition}

\section{Journals}

\section{Édition électronique}

URL : http://journals.openedition.org/leportique/296

DOI : 10.4000/leportique.296

ISSN : $1777-5280$

\section{Éditeur}

Association "Les Amis du Portique"

\section{Édition imprimée}

Date de publication : 1 janvier 1999

ISSN : 1283-8594

\section{Référence électronique}

Pierre-Damien Huyghe, " Art et mécanique », Le Portique [En ligne], 3 | 1999, mis en ligne le 14 mars 2005, consulté le 26 mars 2021. URL : http://journals.openedition.org/leportique/296 ; DOI : https:// doi.org/10.4000/leportique.296

Ce document a été généré automatiquement le 26 mars 2021.

Tous droits réservés 


\title{
Art et mécanique
}

\author{
Pierre-Damien Huyghe
}

1 L'histoire nous apprend que les peintres des temps modernes sont parvenus à faire de leur art, autrefois considéré comme mécanique, un art libéral. Dans cette manière de dire passe l'idée qu'un art - la peinture, procédure majeure de l'image - aurait cessé d'avoir affaire au mécanique après en avoir été partie prenante. C'est même à ce prix qu'elle serait devenue un art éminent. Dans quelle mesure cette affirmation est-elle vraie? Malgré nombre de déclarations théoriques dispersées au long d'une histoire imposante, la dimension libérale des arts de l'image n'est pas si assurée qu'on a pu le croire. Aujourd'hui, il arrive qu'artistes, techniciens et mécaniciens empruntent les mêmes instruments ou les mêmes matériels de travail. Les rapports de l'ingénieur et de l'artiste à la machine et à la machination ne sont pas constamment différents : entre les problèmes qu'il faut résoudre pour mener à bien telle ou telle installation artistique contemporaine et telle ou telle entreprise technologique, il y a parfois bien de la familiarité.

2 Cette familiarité est encore assez difficilement admise. Elle a trouvé dans les déclarations de Baudelaire (Salon de 1859) un adversaire de poids. En s'opposant vigoureusement à l'industrie (et, en même temps, à la photographie ou à l'appareillage photographique de l'image), Baudelaire entendait distinguer l'art, c'est-à-dire, à la fois, le séparer (éviter de le confondre) et l'honorer (lui rendre un culte, le célébrer). Mais le ton même adopté pour cette célébration suffirait à montrer qu'elle n'allait pas de soi et qu'il y avait à lutter contre la plausibilité de la position inverse prête à distinguer la photographie (et, pour Baudelaire, l'industrie) plutôt que la peinture (ou, pour Baudelaire, l'art). Tout se passe comme si Baudelaire craignait de devoir reconnaître (avouer ?) que la différence entre les registres de l'art et du mécanique était en réalité bien ténue. Au reste, il finit par admettre une complémentarité entre les deux: l'industrie, vouée à la reproduction, pouvait se mettre «au service» de l'art pictural comme autrefois l'imprimerie s'était mise « au service » de la poésie.

3 Quel art aujourd'hui se passera d'un tel service ? La diffusion des œuvres l'impose. Mais s'agit-il là seulement d'un service ? À Adorno estimant que le disque, c'est-à-dire la reproduction mécanisée de la musique ${ }^{1}$, sert les œuvres et leur trouve des espaces, 
donc des accès, jusqu'alors inaccessibles, on rétorquera que le format des supports de diffusion aura très vite pesé sur la création elle-même ${ }^{2}$. Le service reproductif exige de l'artiste des décisions préalables à l'œuvre. Par conséquent, il l'oblige. Le rapport est finalement renversé. C'est pour avoir entrevu ce sort mécanique de l'art que Baudelaire aura voulu maintenir le principe de la séparation artistique, quitte, selon ses termes, à « sacrifier le progrès ".

Dans L'CEuvre d'art à l'ère de sa reproduction mécanisée, Walter Benjamin a pris acte du problème et tenté de dégager une position non baudelairienne, c'est-à-dire capable d'accueillir en art les nouveautés de la technique. Cet accueil constituerait un signe de progressisme au regard de Baudelaire. Pour Benjamin, il ne s'agit pas de se résoudre mélancoliquement au sacrifice ${ }^{3}$, mais de trouver ce que peut être le progrès de l'art, ou sa marche, sa méthode, avec l'industrie. Art et industrie devront aller ensemble, sans se sacrifier ni l'un ni l'autre et sans se sacrifier l'un à l'autre. C'est donc le rapport de service de l'industrie (ou de la photographie) à l'art qui fait l'objet de la critique de Benjamin. La possibilité pour le cinéma d'être traité et considéré comme un art est, après la photographie, un enjeu particulier de cette critique.

La position adoptée par Walter Benjamin conduit à l'énoncé de deux thèses. La première constitue l'un des buts explicites de L'Euvre d'art à l'époque de sa reproduction mécanisée. Elle provient de l'idée d'une industrie pensée, au moins partiellement, comme un système historique de diffusion (c'est ainsi que Baudelaire aussi la prend). Un tel système n'a pas toujours eu lieu. C'est donc un fait historique. Dès lors, admettre l'hypothèse d'un art familier de la diffusion industrielle, c'est admettre l'hypothèse d'un art historique différant de lui-même au gré des modes de la diffusion. Le concept gagne donc en diversité, mais perd en unité et en autonomie. L'industrie charrie dans ses formats de diffusion bien autre chose que des œuvres. Son économie autorisera-telle, pour ces dernières, des précautions de traitement particulières ? Le mercantilisme pourrait fort bien avoir raison de ces précautions. Dès lors, comment retrouver les œuvres authentiques parmi toutes les productions diffusées? La possibilité même de cette question aura ouvert une crise des critères et des institutions de repérage artistique. Cette crise est aujourd'hui mal occultée par l'audience des musées: en réalité, elle sourd de l'abondance même de l'offre muséale, de son inquiétude permanente à manquer ce qui risquerait d'être essentiel.

6 La seconde thèse issue de l'analyse benjaminienne est comme enveloppée dans ce qui vient d'être dit : sa formulation demande un retour réflexif sur la démarcation de l'art, c'est-à-dire son indépendance ou sa libéralisation. La méditation de Benjamin serait incompréhensible (et son livre inutile) s'il ne s'avérait pas que l'art est effectivement touché par la reproduction mécanisée des œuvres. D'où, pour nous, cette affirmation : entre art et mécanique la séparation ne s'est pas absolument effectuée, ou ne s'est effectuée qu'apparemment. L'histoire de la libéralisation de la peinture, avec laquelle s'est constituée la grande histoire de l'art (l'histoire des beaux arts), ne donne qu'un aspect de l'histoire réelle, l'officiel ou le glorieux. Mais il est un autre aspect, moins célébré, et qu'il faudra dire dans une histoire plus circonspecte quant à la délimitation de son champ.

7 Ce dernier propos, même s'il n'est pas complètement élaboré chez Benjamin, n'est pas pour autant implicite. Ne le trouve-t-on pas, et très tôt, dans la notion de teneur chosale des œuvres à laquelle pouvait s'opposer, d'un point de vue critique (mais non esthétique ?) la notion de teneur de vérité? Peut-on toutefois séparer effectivement, 
dans l'expérience d'une œuvre, l'une et l'autre teneur? Y a-t-il même une teneur quelconque abstraction faite de la teneur chosale ${ }^{4}$ ?

8 La même question pourrait apparaître dans le projet cher à Régis Debray d'une médiologie. Projet séduisant, annonçant qu'il ne peut rien nous arriver (pas même des pensées) qui ne doive à des appareils. C'est que pour Debray l'être humain est foncièrement mimétique, c'est-à-dire joueur, spectateur, être de re-présentation. Or cela ne lui est pas donné : ce n'est pas génétiquement qu'un homme est dans l'imitation et le spectacle, mais techniquement. Personne ne pourra donc penser sérieusement le rapport de l'humanité à la représentation (ou à la réflexivité) sans impliquer la technicité. L'état des réflexions humaines se lie à celui des techniques. Régis Debray écrit ainsi :

«Doté de la faculté de représenter un comportement par un autre, l'animal hiéroglyphe est imitant autant que parlant; la mimesis serait alors le propre de l'homme, un propre antérieur au logos. La bête simule par intérêt biologique ; c'est la feinte non le jeu. La perdrix fait la blessée pour échapper au prédateur, le loup vaincu tend la gorge, rituel de soumission, pour ne pas mourir. Ni les anges ni les bêtes ne se donnent la comédie. Seul l'homme joue. Le clown, l'acrobate, le danseur, l'équilibriste deviennent en ce cas aussi exemplaire de l'être spirituel de l'homme sa capacité métaphorique - que le poète ou le philosophe. Et c'est une même tradition qui a réputé vilains et indignes le comédien et l'artisan, le mime et l'ingénieur. C'est bien comme imitation ou simulacre que Platon récuse l'objet technique... $»^{5}$.

9 Le problème, c'est que tout cela finit, ou revient, au théâtre. "L'enjeu, écrit encore Debray, porte un nom : la représentation, ou le maintien des distances donneuses de sens ». La médiologie entend donc bien voir subsister (maintenir, dit son auteur) l'idée d'une séparation entre deux mondes à distance l'un de l'autre : d'un côté le monde des acteurs, de l'autre celui des spectateurs, la pratique et la théorie face à face. Et donc séparées. Leurs mondes ne sont pas équivalents : l'un (le symbolique, le théorique où aboutit la représentation) permet de repérer l'autre (le réel où nous vivons), mais sans réciproque. Il y a donc là, en dépit de la critique portée par ailleurs à Platon, comme un arrière monde, une possibilité d'être à distance. De cette possibilité, l'art serait en somme la technique d'accès. Le médium, non la condition. L'inquiétude susceptible de nous saisir à l'évocation de la matérialité d'arts éventuellement mineurs se dissipe à présent: la perfection dont l'espèce est capable doit toujours s'accomplir dans le spectacle de la comédie humaine. Ce spectacle, finalement nostalgique et mélancolique, Régis Debray le pense institué pour un public, non une audience. Son lieu est donc un théâtre, fût-il imaginaire. Au théâtre chacun juge de sa place dans la proximité des autres et ce mode d'appréciation fournit une assez bonne figure des procédures d'élaboration d'une position collective (le théâtre fait du spectacle sur scène une chose publique pour une assemblée des spectateurs). Dans l'audience, tout cela disparaît. Depuis la salle de cinéma, a fortiori avec la télévision puis avec l'informatique, nous n'apercevons plus l'ampleur des côtoiements, nous ne jugeons plus parmi les autres. Le public s'absente du lieu où il pourrait se figurer comme corps constitué et jugeant. Il se dissipe.

Une telle dissipation est sans doute dramatique pour l'amateur de cette particulière machination du public qu'est tout de même un théâtre. Pourtant ce qui vient à manquer d'énergie et à perdre en puissance n'est peut-être qu'un mode de manifestation de l'instance publique. Ce mode est l'assemblée. Lui seul a perdu en rendement. Et c'est à présent une autre machination qui s'élabore. Qui peut déjà 
affirmer que cette nouvelle machination serait intrinsèquement incapable de décliner une nouvelle figure du public et des choses publiques?

11 Pour saisir ce qu'il en est, imaginons un théâtre sans salle. Dans cette configuration hypothétique la scène (le plateau), n'étant plus destinée à un espace de rassemblement, ne pourrait plus se définir que comme l'extrême avancée des actuelles coulisses du théâtre : elle ne serait plus qu'un appareillage de la machinerie, pas tout à fait un espace spectaculaire. La conséquence d'un tel dispositif, ce serait la chute de la tension où pouvait s'alimenter et s'entretenir toute la subjectivité du spectateur seul parmi d'autres et, de là, l'idée même de subjectivité comme être distinct. La disparition de la théâtralité pourrait aussi bien signer la fin d'une illustration et d'une figuration du sujet. Ce dernier s'installait classiquement au théâtre où il se repérait. Il trouvait là un espace démarqué où le lieu des actes (la scène, la coulisse, tous lieux des techniques, des machines et des machinations) se séparait de sa propre résidence (la salle, essentiel lieu de conscience et d'élaboration du goût). La distinction salle/scène recoupait la distinction sujet/monde, théorie/pratique, etc.

C'est là ce que Walter Benjamin met en question sans nostalgie. Ainsi écrivait-il au chapitre 14 de L'Euvre d'art à l'ère de sa reproduction mécanisée :

«La prise de vue et surtout l'enregistrement d'un film offrent une sorte de spectacle telle qu'on n'en avait jamais vue auparavant. Spectacle qu'on ne saurait regarder d'un point quelconque sans que tous les auxiliaires étrangers à la mise en scène même - appareils d'enregistrement, d'éclairage, état-major d'assistants - ne tombent dans le champ visuel (à moins que la pupille du spectateur fortuit ne coïncide avec l'objectif). Ce simple fait suffit seul à rendre superficielle et vaine toute comparaison entre enregistrement au studio et répétition théâtrale. De par son principe, le théâtre connaît le point d'où l'illusion de l'action ne peut être détruite. Ce point n'existe pas vis-à-vis de la scène de film qu'on enregistre. La nature illusionniste du film est une nature au second degré - résultat du découpage. Ce qui veut dire : au studio l'équipement technique a si profondément pénétré la réalité que celle-ci n'apparaît dans le film que grâce à une procédure particulière - à savoir l'angle de prise de vue par la caméra et le montage de cette prise avec d'autres du même ordre. Dans le monde du film la réalité n'apparaît dépouillée des appareils que par le plus grand des artifices et la réalité immédiate s'y présente comme la fleur bleue au pays de la technique ${ }^{6}$.»

Ce texte doute de la plausibilité d'un quelconque naturel pour toute perception qui serait dans l'empire du film. Le point d'illusion au théâtre étant destructible, ce dernier peut être foyer pour une vérité (elle se dégagera par destruction de l'illusion). Mais le film ? En lui disparait le foyer de vérité. Les démarcations où se repère le vrai ne sont pas pertinentes dans le film. Ainsi la distinction entre le naturel (le réel en un sens) et l'artificiel n'a-t-elle pas de sens à l'âge du cinéma. Le cinéma signe le manque de nature. C'est-à-dire encore : le studio figure l'instance à partir de laquelle du réel est possible pour nous. Notre réel est un réel de studio. En dépit de l'artifice extrême qui, au moment de la perception, soustrait la plus grande part de la procédure à la conscience (de sorte que chacun croit décider de ce qu'il voit), nous ne voyons qu'appareillés. Dans ce moment, qui est parfois aussi affaire de salle - proche et cependant si différente du théâtre -, il arrive que nous nous considérions comme des spectateurs. Nous nous imaginons capables de déceler le point d'où tomberaient les illusions, nous rêvons un théâtre encore possible et pertinent. Mais nous sommes en fait au bout du studio, manœuvrés, travaillés, induits par lui, à notre insu évidemment. Nous-mêmes sommes 
l'appareillage ultime de ses appareils. Rien ne nous vient finalement qu'appareillé. Nous devons la modalité de notre être au monde à une logique de studio.

Cette logique, Benjamin ne peut l'étudier sans emprunter au lexique de la dynamique. Cela ne signifie pas qu'il dénie tout intérêt au vocabulaire et aux considérations d'ordre optique impliqués par les arts classiques (sculpture, peinture et théâtre essentiellement). Mais l'optique des hommes à l'époque des arts mécanisés, optique au demeurant complexe (tactile, dit Benjamin, et non seulement visuelle) implique une dynamique, un mouvement, des chocs. L'art du film (le cinéma proprement dit, le cinéma comme art élaboré) apparaît même à Benjamin comme un traitement particulier des chocs. Le champ d'une possible mécanique du film est ainsi indiqué. Définie comme science et non seulement comme pratique, cette mécanique devrait pouvoir énoncer les règles de l'art.

Or ces règles manquent. Il n'y a pas de science de l'art. Et c'est dans ce manque que nous sommes. Aurons-nous un espace de repli où nous réfugier? Un tel espace, pour être refuge, doit être prêt d'avance. D'où l'envie, à nouveau, de ce qui a déjà donné des signes sûrs et rassurants de présence: le théâtre, la peinture classique, les espaces démarqués, optiques, spéculatifs, spectaculaires. Pourtant cette configuration, très renaissante, a cessé de faire effet. Elle ne compte plus que réactivement, dans la fuite et dans la peur. Nous le mesurons aisément en peinture où la double proposition d'Alberti (fonder la peinture en science, définir les lois du dessin d'une part, démarquer théâtralement l'espace du regard d'autre part) a manifestement perdu de sa crédibilité dès l'aube du cinéma. Mais nous pourrons aussi le mesurer en science, dans la mécanique même: qu'il n'y ait pas de science de l'art aura été une affaire de science aussi bien que d'art. Et ce manque nous contraint à envisager comme plausible l'hypothèse d'une révolution: le retour de notre monde dans l'instance des arts mécaniques, dans le manque de science générale, au sens moderne du mot s'entend.

Autrefois, avant la science, le statut des peintres était celui de chirurgiens (littéralement : keir-ergon, ce qui se fait, ou s'organise, à la main). C'est curieusement ce vocabulaire qui vient à l'esprit de Benjamin pour décrire l'opération filmique, l'effet de la logique des studios. Ainsi le texte cité ci-dessus se poursuit-il en ces termes:

«Ces données [du studio], ainsi bien distinctes de celles du théâtre, peuvent être confrontées de manière encore plus révélatrice avec celles de la peinture. Il nous faut ici poser cette question : quelle est la situation de l'opérateur par rapport au peintre? Pour y répondre, nous nous permettons de tirer parti de la situation de l'opérateur, usuelle en chirurgie. Or, le chirurgien se tient à l'un des pôles d'un univers dont l'autre est occupé par le magicien. Le comportement du magicien qui guérit un malade par l'imposition des mains diffère de celui du chirurgien qui procède à une intervention dans le corps du malade. Le magicien maintient la distance naturelle entre le patient et lui ou, plus exactement, s'il ne la diminue par l'imposition des mains - que très peu, il l'augmente - par son autorité - de beaucoup. Le chirurgien fait exactement l'inverse: il diminue de beaucoup la distance entre lui et le patient - en pénétrant à l'intérieur du corps de celui-ci - et ne l'augmente que de peu - par la circonspection avec laquelle sa main se meut parmi les organes. Bref, à la différence du mage (dont le caractère est encore inhérent au praticien), le chirurgien s'abstient au moment décisif d'adopter le comportement d'homme à homme vis à vis du malade : c'est opératoirement qu'il le pénètre plutôt.

Le peintre est à l'opérateur ce que le mage est au chirurgien. Le peintre conserve dans son travail une distance normale vis-à-vis de la réalité de son sujet - par 
contre le cameraman pénètre profondément les tissus de la réalité donnée. Les images obtenues par l'un et par l'autre résultent de procès absolument différents. L'image du peintre est totale, celle du cameraman faite de fragments multiples coordonnés selon une loi nouvelle. C'est ainsi que, de ces deux modes de représentation de la réalité - la peinture et le film - le dernier est pour l'homme actuel incomparablement le plus significatif, parce qu'il obtient de la réalité un aspect dépouillé de tout appareil - aspect que l'homme est en droit d'attendre de l'œuvre d'art - précisément grâce à une pénétration intensive du réel par les appareils. " ${ }^{7}$ naturel, il se présente comme milieu de vie. La situation des hommes dans ce milieu fait penser à la scène montée par Galilée au début du Dialogue sur les sciences nouvelles (et qu'il appelait plutôt lui-même Traité du mouvement). Tels les ouvriers enfermés dans les arsenaux, ouvriers que cette scène désigne et nomme mais ne montre pas, nous serions dotés "d'une expérience remarquable et d'un jugement des plus subtils », usant et vivant d'instruments et machines prodigieux, mais " oiseux » et guère judicieux dans nos explications (à la vérité, nous serions même incapables d'élaborer une véritable théorie de nos faits et gestes).

18 Ce qui sauve l'humanité, chez Galilée, c'est une parole venue du dehors. Cette parole s'ancre, comme chez Alberti le dessin, dans la géométrie. On ne sait si cette géométrie est pure. Mais son recours permet d'énoncer «les lois abstraites et idéales de la mécanique ». Il appartient à la science (alors nouvelle, la science des temps modernes) d'assigner les pratiques mécaniques à une telle idéalité législatrice. Cette entreprise présuppose elle-même très vite (à la première démonstration) sa propre possibilité, se bouclant sur soi selon une procédure logique qui n'a guère à envier aux allégations induites en ce siècle par le ready-made de Duchamp. C'est cette procédure que reprendra Kant au moment de fonder l'entreprise critique : que faut-il dire, étant donné la science ? C'est-à-dire, de manière plus développée : quelle est l'hypothèse nécessaire à l'énoncé d'une proposition scientifique donnée? Ou, dans le cas Galilée : quel est l'énoncé à partir duquel il y a sens à parler hors de l'arsenal, quelle est la proposition susceptible de rapatrier la pensée sur un lieu théorique capable de fournir un siège à l'esprit (ou d'instituer, pour ainsi dire, son théâtre) et de donner à voir le monde comme une scène spectaculaire?

19 Cette proposition se résume dans la phrase selon laquelle « en fin de compte, à toute machine ou construction non seulement artificielle mais encore naturelle, est nécessairement assignée une limite que ni l'art ni la nature ne sauraient outrepasser... ${ }^{8}$.

Que vaut à présent cette phrase? Quelle est sa plausibilité ? La nature n'est-elle pas elle-même un effet spécial, un mode des techniques et de l'art ? Depuis le film au moins, nous pouvons penser que nous sommes dans un monde d'artistes. Nous vivons dans l'arsenal. Nous ne percevons donc pas naturellement, nous ne voyons et sentons que mécaniquement. Dès lors, il n'est pas de terme de comparaison indépendant (la « construction naturelle » dans le texte de Galilée) à quoi rapporter les constructions artistiques. D'où l'idée que ces constructions se rapportent en fait sans cesse à ellesmêmes (à leur propre excitation interne), en automate.

21 Cette hypothèse d'une existence humaine prise dans une mécanique généralisée correspond à une antique hantise. Elle justifie, depuis Platon, le recours à toutes sortes de transcendances : il n'y aurait de sagesse (et de philosophie) possible qu'à partir d'un point de vue extérieur aux procédures mécaniques. Celles-ci doivent donc être 
" assignées à une limite ", c'est-à-dire liées à des fonctions et à des territoires, intégrées dans des domaines recensables de possibilités. L'élaboration de semblables liaisons impliquera volontiers le point de vue surplombant d'un créateur ou la perspective d'un théoricien : ces deux êtres auront en commun de voir à distance et d'avance. De là, il semble que le nom de mécanique convient à l'inverse à ce qui en soi est procédure de dé-limitation, modalité d'affranchissement des géographies conceptuelles. En soi, donc, les théoriciens l'ont toujours secrètement su, le mécanique ne s'assignerait pas. D’ailleurs, il ignore la réflexivité, il ne se distancie pas.

L'originalité philosophique de Benjamin est d'envisager, à propos du film, et au titre de la sagesse, une conduite de distanciation. Il s'agit en somme pour lui d'espacer la mécanique du film, non de l'évaluer du dehors. Un tel espacement desserrerait en quelque sorte la machination du monde où nous nous trouvons. Mais la perspective, le point de vue à distance manquent ici a priori. Ce ne sont pas des données transcendantales. À l'époque de la mécanique généralisée, il n'y a pas de résidence subjective autonome, pas d'être à soi du sujet, pas de perspective contemplative, pas de théorie par conséquent. Ou bien, si tout cela a lieu, c'est à titre de refuge, dans l'imaginaire.

Comment penser et nommer l'espacement auquel invite la réflexion de Benjamin ? Quelle est sa condition de possibilité? Quelle puissance l'autorise? Ces questions ne peuvent être traitées à partir d'un doute sur leur plausibilité. Il nous faut, à l'exemple méthodique de Kant, présupposer la possibilité d'une réponse. Sinon, je le répète, l'imaginaire nous guette pour repos.

Donnons-nous donc l'espacement. Et pour cela, d'abord, partons du film (et non du théâtre) et de ce qui donne le film lui-même : le studio, la caméra, l'appareil de prise de vue. Notre question, aussitôt, se précise : nous comprenons que nous sommes en train de chercher la procédure d'une prise de vue (ou d'un filmage) s'espaçant. Cette question a de multiples aspects: philosophique (elle implique des recherches sur la possibilité même de l'espacement, sur ce qui le rend pensable), institutionnel (il y a lieu de chercher dans quel mode de travail peut s'espacer l'univers du film), technique (une manœuvre particulière doit se mettre en place), etc. Faisons l'hypothèse que l'ensemble de ces recherches permettrait de définir la condition de l'art contemporain, c'est-àdire, dans le langage de Walter Benjamin, de l'art à l'époque du film ou de la caméra. J'ajouterais ces questions, qui ne tombent pas immédiatement sous le propos de Benjamin : qu'y peut la peinture? Que peut un peintre au temps des cameramen? Quelle est la responsabilité possible d'une peinture contemporaine du film? Quelle est même sa visibilité ? Au reste, qu'est-ce qui peut être vu, et qu'est-ce que regarder, dans le monde du cinéma?

L'hypothèse la plus probable, c'est que la peinture soit amenée à échapper à l'histoire qui a fait d'elle un art libéral, c'est-à-dire un art à l'allure dégagée du mécanique, un art du génie. Nombre de peintres ont aujourd'hui quitté la peinture. Ils en sont en fait à déceler ce qui, dans la peinture illustre des Temps Modernes, était demeuré à l'écart du spectacle, pratique obscure ou négligée. Les noms ne manquent pas de ces artistes qui, de la projection à la lumière ou à la prise de vue, exposent peu à peu dans les conditions de la technique contemporaine ce que la peinture grandie par le mouvement de libéralisation avait tantôt ignoré, tantôt sublimé, mais jamais véritablement accepté. Ainsi l'art se travaille-t-il lui-même. Sur le chemin de ce travail, la nostalgie de la tradition délaissée du tableau est toujours possible. Mais ce qui peut aussi bien 
apparaître, de manière finalement plus joyeuse, c'est un art effectivement devenu contemporain : un art à jour.

\section{NOTES}

1.. Le thème de la reproduction mécanisée de l'œuvre d'art a focalisé, comme on sait, la réflexion de Walter Benjamin dont nous allons parler. Le texte qui en est issu, L'Euvre d'art à l'époque de sa reproduction mécanisée, est fort connu et commenté. On connait au moins un texte de Valéry dont l'existence suffit à montrer que le problème appartient à l'époque plutôt qu'à tel ou tel penseur. Ce texte, du reste évoqué par Benjamin, est intitulé La Conquête de l'ubiquité. Il admet l'idée d'une « technique des arts » qui serait «transformée » par de "grandes nouveautés » capables d'« agir sur l'invention ellemême » et de «modifier merveilleusement la notion même d'art ». Valéry ajoute : « le travail de l'artiste musicien, auteur ou virtuose, trouve dans la musique enregistrée la condition essentielle du rendement esthétique le plus haut ». Et il conclut sur "l'intimité nouvelle de la Musique avec la Physique, dont l'alliance immémoriale nous avait déjà tant donné ".

2. Il conviendrait de porter une attention toute particulière aux problèmes de format et de formatage. Une opération artistique est-elle possible sans format? Ne consiste-telle pas précisément, en un sens étrange du mot, à dé-limiter un format, c'est-à-dire à le pousser, à le détourner, à lui faire prendre un passage ? En tout état de cause, il n'y aurait pas d'œuvre qui ne soit une particulière pesée ou considération du format à partir duquel elle a lieu.

3.. Cette expression mériterait une défense particulière compte tenu de ce qui se dit par ailleurs au sujet de Benjamin. On se reportera en particulier au indications données en quatrième de couverture par Gérard RAULET dans son récent livre, Le Caractère destructeur. Esthétique, théologie et politique chez Walter Benjamin, Aubier, 1997. Cet ouvrage « constate que l'œuvre de Benjamin est le document d'un échec » ou encore " qu'elle n'a rien de plus à nous apprendre que son échec ». C'est à voir.

4.. La distinction entre teneur chosale et teneur de vérité est élaborée par Walter BENJAMIN dans «Les Affinités électives » de Goethe, texte rédigé en 1922 et publié pour la première fois en 1924-25. Une traduction de ce texte, due à Maurice de Gandillac, se trouvait dans le recueil aujourd'hui introuvable des Euvres de Benjamin, tome 1, « Mythe et violence».

5.. Régis DEBRAY, « Pourquoi le spectacle? ", in Les Cahiers de médiologie, $\mathrm{n}^{\circ} 1$, Gallimard, 1996, p. 11.

6.. BENJAMIN, «L'œuvre d'art à l'époque de sa reproduction mécanisée », in Écrits français, Gallimard, 1991, p. 159-160. Le texte est souligné par Benjamin lui-même. 7.. BENJAMIN, op. cit., p. 160-161. Le texte est de nouveau souligné par Benjamin luimême.

8.. GALILÉE, « Dialogue sur les sciences nouvelles », in Dialogues et lettres choisies, Hermann, 1966, p. 227. 